\title{
ON THE HÖLDER CONTINUITY OF SOLUTIONS OF A CERTAIN SYSTEM RELATED TO MAXWELL'S EQUATIONS
}

\author{
KYUNGKEUN KANG AND SEICK KIM
}

\begin{abstract}
In this paper, we prove the Hölder continuity of weak solutions of a certain system arising from the Maxwell's equations in a quasi-stationary electromagnetic field.
\end{abstract}

\section{INTRODUCTION}

Let $\Omega$ be a domain in $\mathbb{R}^{3}$ and $a \in L^{\infty}(\Omega)$ be a scalar function bounded by two positive numbers $m, M$. In this paper we study the regularity problem of the following system:

$$
\left.\begin{array}{c}
\nabla \times[a(x) \nabla \times u]=f+\nabla \times g \\
\nabla \cdot u=0
\end{array}\right\} \quad \text { in } \Omega .
$$

Here we denote $\nabla \times u=\operatorname{curl} u$ and $\nabla \cdot u=\operatorname{div} u$. The question about regularity of solution of such system was raised by Professor M. Giaquinta. The main result of this paper is Hölder continuity of weak solutions of system (1.1) under appropriate assumptions on the inhomogeneous terms $f, g$.

The above system arises from Maxwell's equations in a quasi-stationary electromagnetic field where the displacement of electrical current is assumed to be time independent. We are grateful to Professor M. Hong for valuable discussions elucidate the connection to Maxwell equations. In the study of the penetration of a magnetic field in materials, the electrical resistance strongly depends on the temperature and by taking the temperature effect into consideration the classical Maxwell system in a quasi-stationary electromagnetic field reduces to the following mathematical model: find $H(x, t)$ and $u(x, t)$ such that

$$
\left\{\begin{array}{l}
H_{t}+\nabla \times[\sigma(u) \nabla \times H]=0, \\
\nabla \cdot H=0, \\
u_{t}-\Delta u=\sigma(u)|\nabla \times H|^{2},
\end{array}\right.
$$

where $H$ and $u$ represents, respectively, the strength of the magnetic field and temperature while $\sigma(u)$ denotes the electrical resistivity of the material (see e.g. [9]). In particular, in the steady-state we have the following steady-state system:

$$
\left\{\begin{array}{l}
\nabla \times[\sigma(u) \nabla \times H]=0, \\
\nabla \cdot H=0, \\
-\Delta u=\sigma(u)|\nabla \times H|^{2} .
\end{array}\right.
$$

Global existence of a pair of weak solutions $(H, u)$ of the system (1.2) was established by YIN [9]. However, the continuity of weak solutions of the system (1.2)

The first author was supported in part by NSF Grant No. DMS-9877055.

The second author was supported in part by NSF Grant No. DMS-9971052. 
as well as the system (1.3) is unknown. In Sec. 3, we will show that, by using our result on the linear system (1.1), weak solutions of the coupled nonlinear system (1.3) are locally Hölder continuous.

As we mentioned earlier, the motivation for studying the system (1.1) is an interesting question which has been raised by GIAQUINTA \& Hong [4]. The original formulation of the question appears in terms of differential forms. However, in the case when $n=3$, it can be rephrased as follows: Are weak solutions to the following system locally Hölder continuous?

$$
\left.\begin{array}{c}
\nabla \times[a(x) \nabla \times u]=0 \\
\nabla \cdot u=0
\end{array}\right\} \quad \text { in } \quad \Omega
$$

Indeed, the above system (1.4) is a special case of (1.1) and as we mentioned at the beginning the answer is positive when $n=3$. We don't know the answer for higher dimensions $n \geq 4$. In Sec. 4 we will formulate their original question by using differential forms and discuss some related problems. Very recently, we received a preprint by Professor H. Yin [10], in which a similar result to ours is obtained. It seems [10] used an idea similar to ours, although technical details are different.

\section{Main Results: Hölder estimates}

In this section we shall always assume $n=3$. First, we will introduce notations.

- For $x \in \mathbb{R}^{n}$ and $\rho>0$ we define $B_{\rho}(x)=\left\{y \in \mathbb{R}^{n}:|x-y|<\rho\right\}$.

- For a measurable set $S \subset \mathbb{R}^{n}$, we define $\oint_{S} f=\frac{1}{S} \int_{S} f d x$.

- We denote $(f)_{x, \rho}=f_{x, \rho}=f_{B_{\rho}(x)} f d x$.

- We denote $B_{\rho}=B_{\rho}(x)$ and $f_{\rho}=f_{x, \rho}$ if $x$ is clear from the context.

- Let $\mathcal{D}(\Omega)=\mathcal{D}\left(\Omega ; \mathbb{R}^{n}\right)=\left\{f \in C^{\infty}\left(\Omega ; \mathbb{R}^{n}\right): \nabla \cdot f=0\right\}$. We denote by $\mathcal{H}^{q}(\Omega), q \in[1, \infty)$, the completion of $\mathcal{D}(\Omega)$ in the norm of $L^{q}(\Omega)$.

- $\Omega^{\prime} \Subset \Omega$ means $\Omega^{\prime}$ is a precompact subset of $\Omega$.

- For $u=\left(u^{1}, \cdots, u^{n}\right)$, we denote by $\nabla u$ the gradient matrix: $(\nabla u)_{i j}=D_{j} u^{i}$.

Now, we will state our main results. Consider the following linear system:

$$
\left.\begin{array}{c}
\nabla \times[a(x) \nabla \times u]=f \\
\nabla \cdot u=0
\end{array}\right\} \quad \text { in } \quad \Omega
$$

where $f \in \mathcal{H}^{q}\left(\Omega ; \mathbb{R}^{n}\right)$ and $a \in L^{\infty}(\Omega ; \mathbb{R})$ such that $m \leq a \leq M$ for some constants $m, M>0$. The restriction $f \in \mathcal{H}^{q}\left(\Omega ; \mathbb{R}^{n}\right)$ arises from the consistency condition

$$
0=\nabla \cdot \nabla \times[a(x) \nabla \times u]=\nabla \cdot f
$$

in the sense of distribution.

Theorem 2.1. Let $u \in W_{\text {loc }}^{1,2}\left(\Omega ; \mathbb{R}^{n}\right)$ be a weak solution of the system (2.1) with $f \in \mathcal{H}_{l o c}^{q}(\Omega), q>n / 2$. Let $B:=B_{R}\left(x_{0}\right) \Subset \Omega$. Then there exists constants $\alpha=\alpha(m, M, q)>0$ and $C=C(m, M, q, R)$ such that $u$ is Hölder continuous in $B_{R / 16}\left(x_{0}\right)$ and

$$
\|u\|_{C^{0, \alpha}\left(\bar{B}_{R / 16}\right)} \leq C\left[\|u\|_{L^{2}(B)}+\|f\|_{L^{q}(B)}\right]
$$

Next, we consider the quasi-linear system:

$$
\left.\begin{array}{c}
\nabla \times[\sigma(x, u) \nabla \times u]=f \\
\nabla \cdot u=0
\end{array}\right\} \quad \text { in } \Omega .
$$

Here we assume $f \in \mathcal{H}_{l o c}^{q}(\Omega)$ and $\sigma: \Omega \times \mathbb{R}^{n} \rightarrow \mathbb{R}$ satisfies the following conditions: 
(a) $m \leq \sigma \leq M$ for positive constants $m, M$.

(b) $\sigma$ is Hölder continuous in $\Omega \times \mathbb{R}^{n}:[\sigma]_{\mu}=[\sigma]_{C^{0, \mu}\left(\Omega \times \mathbb{R}^{n}\right)}<\infty$, for some $\mu \in(0,1)$.

Theorem 2.2. Let $u \in W_{\text {loc }}^{1,2}\left(\Omega ; \mathbb{R}^{n}\right)$ be a weak solution of (2.3) and assume $f \in$ $\mathcal{H}_{\text {loc }}^{p}(\Omega), p>n$. Let $B:=B_{R}\left(x_{0}\right) \Subset \Omega$. Then, under above assumptions on $\sigma, \nabla u$ is locally Hölder continuous with exponent $\alpha=\min (\mu, 1-n / p)$ and

$$
\|u\|_{C^{1, \alpha}\left(\bar{B}_{R / 4}\right)} \leq C, \quad C=C\left(m, M, p,[\sigma]_{\mu},\|u\|_{W^{1,2}(B)},\|f\|_{L^{p}(B)}, R\right) .
$$

The following technical lemmas will be used in the proof of theorems.

Lemma 2.1 (Uniqueness). Let $u \in W_{0}^{1,2}(\Omega)$ be a weak solution of

$$
\left.\begin{array}{c}
\nabla \times[a(x) \nabla \times u]=0 \\
\nabla \cdot u=0
\end{array}\right\} \quad \text { in } \Omega .
$$

Then $u \equiv 0$ in $\Omega$.

Proof. Since $\nabla \cdot u=0$, integration by parts yields

$$
\int_{\Omega}|\nabla \times u|^{2}=\int_{\Omega}|\nabla \times u|^{2}+|\nabla \cdot u|^{2}=\int_{\Omega}|\nabla u|^{2} .
$$

On the other hand, by using $u$ itself as a test function we have

$$
m \int_{\Omega}|\nabla \times u|^{2} \leq \int_{\Omega} a(x)|\nabla \times u|^{2}=\int_{\Omega} \nabla \times[a(x) \nabla \times u] \cdot u=0 .
$$

Hence, $\nabla u=0$ in $\Omega$. This completes the proof.

Lemma 2.2. Let $B \subset \mathbb{R}^{n}$ be a open ball and let $f \in \mathcal{D}(B)$. Then there exists $g \in C^{\infty}\left(B ; \mathbb{R}^{n}\right) \cap \mathcal{D}(B)$ such that $\nabla \times g=f$ in $B$ and $g=0$ on $\partial B$. Moreover, if $f \in \mathcal{H}^{p}(B), 1<p<\infty$, then $\|\nabla g\|_{L^{p}(B)} \leq C(p)\|f\|_{L^{p}(B)}$.

Proof. Let $g$ be the unique solution of

$$
\left\{\begin{array}{ccc}
-\Delta g=\nabla \times f & \text { in } \quad B \\
g=0 & \text { on } \quad \partial B .
\end{array}\right.
$$

From the following vector identity,

$$
\nabla \times(\nabla \times f)=\nabla(\nabla \cdot f)-\Delta f
$$

and the representation formula of $g$ in terms of the Green's function, it is easy to see $\nabla \times g=f$ and $\nabla \cdot g=0$ in $B$. The second part of lemma follows from $L^{p}$-theory of Laplace operator.

Lemma 2.3. Suppose $F \in C^{\infty}\left(B ; \mathbb{R}^{n}\right)$ satisfy $\nabla \times F=0$ in $B$. Then there exists $\varphi \in C^{\infty}(B ; \mathbb{R})$ such that $\nabla \varphi=F$ in $B$ and $\varphi=0$ on $\partial B$. Moreover, if $F \in L^{2}(B)$, then $\|\varphi\|_{L^{2}} \leq C\|F\|_{L^{2}}$.

Proof. Let $\varphi$ be the unique solution of

$$
\left\{\begin{array}{cc}
\Delta \varphi=\nabla \cdot F & \text { in } \quad B \\
\varphi=0 & \text { on } \quad \partial B .
\end{array}\right.
$$

Then $\nabla \varphi=F$ will follow immediately from Lemma 2.3. Also, $\|\nabla \varphi\|_{L^{2}} \leq C\|F\|_{L^{2}}$. Since $\varphi=0$ on $\partial B$, we can use Poincaré inequality to get $\|\varphi\|_{L^{2}(B)} \leq C\|F\|_{L^{2}}$. 
Lemma 2.4. Let $w \in W_{0}^{1,2}\left(B_{R} ; \mathbb{R}^{n}\right)$ be a weak solution of

$$
\left.\begin{array}{c}
\nabla \times(\nabla \times w)=\nabla \times(F+G+H) \\
\nabla \cdot w=0
\end{array}\right\} \quad \text { in } \quad B_{R}
$$

where $F \in C^{0, \mu}\left(B_{R}\right), \mu>0, G \in L^{2}\left(B_{R}\right)$ and $H \in L^{q}\left(B_{R}\right), q>n$. Then

$\int_{B_{R}}|\nabla w|^{2} \leq C\left([F]_{\mu}^{2} R^{n+2 \mu}+\|G\|_{L^{2}}^{2}+\|H\|_{L^{q}}^{2} R^{n-2+2 \gamma}\right), \quad \gamma=1-n / q>0$.

Proof. From the identity (2.4), $w \in W_{0}^{1,2}$ is a weak solution of

$$
-\Delta w=\nabla \times(F+G+H) \text { in } B_{R} .
$$

By using $w$ itself as a test function we get

$$
\int_{B_{R}} \nabla w \cdot \nabla w=\int_{B_{R}}\left(F-F_{R}\right) \cdot \nabla \times w+\int_{B_{R}}(G+H) \cdot \nabla \times w .
$$

Hence, Schwarz inequality yields

$$
\int_{B_{R}}|\nabla w|^{2} \leq \int_{B_{R}}\left|F-F_{R}\right|^{2}+\int_{B_{R}}|G|^{2}+\int_{B_{R}}|H|^{2}+\frac{3}{4} \int_{B_{R}}|\nabla \times w|^{2} .
$$

Since $\nabla \cdot w=0$, integration by parts yields

$$
\int_{B_{R}}|\nabla \times w|^{2}=\int_{B_{R}}|\nabla \times w|^{2}+|\nabla \cdot w|^{2}=\int_{B_{R}}|\nabla w|^{2} .
$$

The lemma follows from obvious inequalities $\int_{B_{R}}\left|F-F_{R}\right|^{2} \leq C(n)[F]_{\mu}^{2} R^{n+2 \mu}$ and $\|H\|_{L^{2}}^{2} \leq\|H\|_{L^{q}}^{2}\left|B_{R}\right|^{1-2 / q}$.

Lemma 2.5. Let $u \in W^{1,2}\left(B_{2} ; \mathbb{R}^{n}\right)$ be a weak solution of

$$
\begin{gathered}
\nabla \times[a(x) \nabla \times u]=\nabla \times g \text { in } \Omega . \\
\text { Then }\|\nabla \times u\|_{L^{2}\left(B_{1}\right)} \leq C\left(\|u\|_{L^{2}\left(B_{2}\right)}+\|g\|_{L^{2}\left(B_{2}\right)}\right) .
\end{gathered}
$$

Proof. This is a Caccioppoli type inequality. The proof is straightforward.

Lemma 2.6. Let $\phi(t)$ be a nonnegative and nondecreasing function. Suppose that

$$
\phi(\rho) \leq A\left[\left(\frac{\rho}{r}\right)^{\alpha}+\varepsilon\right] \phi(r)+B r^{\beta}
$$

for all $\rho<r \leq R_{0}$, with $A, \alpha, \beta$ nonnegative constants, $\beta<\alpha$. Then there exists a constant $\varepsilon_{0}=\varepsilon_{0}(A, \alpha, \beta)$ such that if $\varepsilon<\varepsilon_{0}$, for all $\rho<r \leq R_{0}$ we have

$$
\phi(\rho) \leq c\left[\left(\frac{\rho}{r}\right)^{\beta} \phi(r)+B \rho^{\beta}\right]
$$

where $c$ is a constant depending on $\alpha, \beta, A$.

Proof. See [3] Lemma 2.1, page 86.

Proof of Theorem 2.1. First, we shall assume that $a \in L^{\infty}(\Omega) \cap C^{\infty}(\Omega)$ and $f \in$ $\mathcal{H}_{l o c}^{q}(\Omega) \cap C^{\infty}(\Omega)$. The constant $C$ which appears in (2.2) will not depend on extra smoothness of data. Since (2.1) is a linear system, the full result will then follow from Lemma 2.1 and standard approximation argument. Also, we will assume without loss of generality that $B=B_{16}\left(x_{0}\right)$. Moreover, we may assume $n / 2<q<$ $n$. The case $q \geq n$ will be recovered by Hölder's inequality. 
Since $\nabla \cdot f=0$, we conclude from Lemma 2.2 that there exists $g \in C^{\infty}\left(B_{8} ; \mathbb{R}^{n}\right)$ such that $f=\nabla \times g$ and $g=0$ on $\partial B_{8}$. Then Sobolev-Poincaré inequality implies

$$
\|g\|_{L^{q^{*}\left(B_{8}\right)}} \leq C\|\nabla g\|_{L^{q}\left(B_{8}\right)} \leq C\|f\|_{L^{q}\left(B_{8}\right)}, \quad q^{*}=n q /(n-q)>n .
$$

Then by Lemma 2.3 there exists $\varphi \in C^{\infty}\left(B_{8} ; \mathbb{R}\right)$ such that

$$
\nabla \varphi=a(x) \nabla \times u-g
$$

and

$$
\|\varphi\|_{L^{2}\left(B_{8}\right)} \leq C\left(\|\nabla \times u\|_{L^{2}\left(B_{8}\right)}+\|g\|_{L^{2}\left(B_{8}\right)}\right) .
$$

From Lemma 2.5 and (2.5), we can estimate $\|\varphi\|_{L^{2}\left(B_{8}\right)}$ in (2.7)

$$
\|\varphi\|_{L^{2}\left(B_{8}\right)} \leq C\left(\|u\|_{L^{2}(B)}+\|f\|_{L^{q}(B)}\right) .
$$

By rewriting (2.6) as $\nabla \times u=a^{-1} \nabla \varphi+a^{-1} g$ we conclude

$$
0=\nabla \cdot(\nabla \times u)=\nabla \cdot\left[a^{-1} \nabla \varphi\right]+\nabla \cdot\left(a^{-1} g\right) .
$$

Now we have a single elliptic equation

$$
-\nabla \cdot\left[a^{-1} \nabla \varphi\right]=\nabla \cdot\left(a^{-1} g\right) .
$$

It is well known that the following estimate holds:

$$
\|\varphi\|_{C^{0, \beta}\left(B_{4}\right)} \leq C\left(\|\varphi\|_{L^{2}\left(B_{8}\right)}+\|g\|_{L^{q^{*}}\left(B_{8}\right)}\right),
$$

where $C=C(M / m, q)$ and $\beta=\beta(M / m)>0$ (see e.g. [5] Theorem 8.24).

Also, from (2.9) we have the following Caccioppoli inequality: for all $r \leq 4$

$$
\begin{aligned}
\int_{B_{r / 2}}|\nabla \varphi|^{2} & \leq C\left(\frac{1}{r^{2}} \int_{B_{r}}\left|\varphi-(\varphi)_{r}\right|^{2}+\int_{B_{r}}|g|^{2}\right) \\
& \leq C\left([\varphi]_{C^{0, \beta}\left(B_{4}\right)}^{2} r^{n-2+2 \beta}+\|g\|_{L^{q^{*}\left(B_{8}\right)}}^{2} r^{n-2+2 \gamma}\right)
\end{aligned}
$$

where $C=C(M / m)$ and $\gamma=\left(2-\frac{n}{q}\right)>0$.

Since $\nabla \cdot u=0,(2.4)$ implies

$$
-\Delta u=\nabla \times(\nabla \times u)=\nabla \times\left(a^{-1} \nabla \varphi\right)+\nabla \times\left(a^{-1} g\right) .
$$

Fix $r \leq 2$ and decompose $u$ into two functions $v$ and $w:=u-v$ such that $v$ is the unique solution of

$$
\left\{\begin{array}{c}
-\Delta v=0 \quad \text { in } \quad B_{r} \\
v=u \quad \text { on } \quad \partial B_{r} .
\end{array}\right.
$$

Then, $w=0$ on $\partial B_{r}$ and solves

$$
-\Delta w=\nabla \times\left(a^{-1} \nabla \varphi\right)+\nabla \times\left(a^{-1} g\right) \text { in } B_{r} .
$$

Hence, from (2.11) and Lemma 2.4 (with $G=a^{-1} \nabla \varphi$ and $H=a^{-1} g$ ) together with Poincaré inequality, we get

$$
\int_{B_{r}}\left|w-w_{r}\right|^{2} \leq C\left([\varphi]_{C^{0, \beta}\left(B_{4}\right)}^{2} r^{n+2 \beta}+\|g\|_{L^{q^{*}\left(B_{8}\right)}}^{2} r^{n+2 \gamma}\right) .
$$


Then, since $v$ is harmonic, the following estimates hold for all $\rho<r \leq 2$ :

$$
\begin{aligned}
\int_{B_{\rho}}\left|u-u_{\rho}\right|^{2} & \leq C\left(\frac{\rho}{r}\right)^{n+2} \int_{B_{r}}\left|u-u_{r}\right|^{2}+C \int_{B_{r}}\left|w-w_{r}\right|^{2} \\
& \leq C\left(\frac{\rho}{r}\right)^{n+2} \int_{B_{r}}\left|u-u_{r}\right|^{2} \\
& +C\left([\varphi]_{C^{0, \beta}\left(B_{4}\right)}^{2} r^{n+2 \beta}+\|g\|_{L^{q^{*}\left(B_{8}\right)}}^{2} r^{n+2 \gamma}\right),
\end{aligned}
$$

where $C=C(m, M)$.

Let $\phi(\rho):=\int_{B_{\rho}}\left|u-u_{\rho}\right|^{2}$ and $\alpha=\min (\beta, \gamma)$. Combining (2.5), (2.8) and (2.10),

$$
\phi(\rho) \leq C\left[\left(\frac{\rho}{r}\right)^{n+2} \phi(r)+r^{n+2 \alpha}\left(\|u\|_{L^{2}(B)}^{2}+\|f\|_{L^{q}(B)}\right)\right] .
$$

Since (2.13) holds for any $\rho<r \leq 2$, by Campanato's integral characterization of Hölder continuous function together with Lemma 2.6, we conclude

$$
[u]_{C^{0, \alpha}\left(B_{2}\right)} \leq C(m, M, q)\left(\|u\|_{L^{2}(B)}+\|f\|_{L^{q}(B)}\right) .
$$

Fix $x \in \bar{B}_{1}$ and consider a ball $B_{1}(x) \subset B_{2}$. Then

$$
|u(x)| \leq|u(y)|+|u(x)-u(y)| \leq|u(y)|+[u]_{C^{0, \alpha}\left(B_{2}\right)}, \quad \forall y \in B_{1}(x) .
$$

Integrating (2.15) with respect to $y$ over $B_{1}(x)$ we get

$$
|u(x)| \leq C\left(\|u\|_{L^{2}\left(B_{2}\right)}+[u]_{C^{0, \alpha}\left(B_{2}\right)}\right), \quad \forall x \in B_{1} .
$$

Combining (2.14), (2.16) we finally obtain

$$
\|u\|_{C^{0, \alpha}\left(\bar{B}_{1}\right)} \leq C(m, M, q)\left(\|u\|_{L^{2}(B)}+\|f\|_{L^{q}(B)}\right) .
$$

This completes the proof.

For the proof of Theorem 2.2, we need $C^{1, \alpha}$-estimates of the linear system (2.1) under the assumption that $a$ is Hölder continuous.

Lemma 2.7. Let $u \in W_{\text {loc }}^{1,2}\left(\Omega ; \mathbb{R}^{n}\right)$ be a weak solution of (2.1) where $f \in \mathcal{H}_{\text {loc }}^{p}(\Omega)$ for some $p>n$. Assume further that $a \in C^{0, \mu}(\Omega ; \mathbb{R})$. Then if $B:=B_{R}\left(x_{0}\right) \Subset \Omega$, $\nabla u$ is Hölder continuous in $B_{R / 4}\left(x_{0}\right)$ and

$$
[\nabla u]_{C^{0, \alpha}\left(B_{R / 4}\right)} \leq C\left(\|\nabla u\|_{L^{2}(B)}+\|f\|_{L^{q}(B)}\right),
$$

Here, $\alpha=\min (1-n / p, \mu)$ and $C=C\left(n, m, M, p,[a]_{\mu}, R\right)$.

Proof. The proof relies on the standard perturbation method. As in Theorem 2.1, we may assume that $f$ is smooth and $B=B_{4}\left(x_{0}\right)$. Then, by Lemma 2.2 there exists $g$ such that $f=\nabla \times g$ and $[g]_{C^{0, \nu}(B)} \leq C\|\nabla g\|_{L^{p}(B)} \leq C\|f\|_{L^{p}(B)}, \nu=1-n / p>0$. Let $y \in \bar{B}_{2}(0)$ and let $R_{0} \leq 2$ be a fixed number which will be specified later. Then

$$
a(y)[\nabla \times(\nabla \times u)]=\nabla \times([a(y)-a(x)] \nabla \times u)+\nabla \times g \text { in } B_{R_{0}}(y) \subset B .
$$

Fix an $r \leq R_{0}$ and split $u$ into $v$ and $w:=u-v$ such that

$$
\left\{\begin{array}{ccc}
-\Delta v=0 & \text { in } \quad B_{r}(y) \\
v=u & \text { on } & \partial B_{r}(y)
\end{array}\right.
$$

Then, $w \in W_{0}^{1,2}\left(B_{r}(y)\right)$ and satisfies

$$
-a(y) \Delta w=\nabla \times([a(y)-a(x)] \nabla \times u+g) \quad \text { in } \quad B_{r}(y) .
$$


Hence from Lemma 2.4 with $F=g$ and $G=[a(y)-a(x)] \nabla \times u$, we obtain

$$
\begin{aligned}
\int_{B_{r}(y)}|\nabla w|^{2} & \leq C\left([a]_{\mu}^{2} r^{2 \mu}\|\nabla \times u\|_{L^{2}\left(B_{r}(y)\right)}^{2}+[g]_{\nu}^{2} r^{n+2 \nu}\right) \\
& \leq C\left(r^{2 \mu} \int_{B_{r}(y)}|\nabla u|^{2}+\|f\|_{L^{p}(B)}^{2} r^{n+2 \nu}\right)
\end{aligned}
$$

Since $\nabla v$ is harmonic in $B_{r}(y)$, the following estimate holds for $\rho<r \leq R_{0}$ :

$$
\begin{aligned}
\int_{B_{\rho}(y)}|\nabla u|^{2} & \leq C\left[\left(\frac{\rho}{r}\right)^{n} \int_{B_{r}(y)}|\nabla u|^{2}+\int_{B_{r}(y)}|\nabla w|^{2}\right] \\
& \leq C\left[\left(\frac{\rho}{r}\right)^{n}+r^{2 \mu}\right] \int_{B_{r}(y)}|\nabla u|^{2}+C\|f\|_{L^{p}(B)}^{2} r^{n+2 \nu} .
\end{aligned}
$$

We will apply Lemma 2.6 to the quantity $\phi(\rho):=\int_{B_{\rho}(y)}|\nabla u|^{2}$. Choose $R_{0}$ small enough so that $R_{0}^{2 \mu}<\varepsilon_{0}$. Then Lemma 2.6 implies

$$
\int_{B_{\rho}(y)}|\nabla u|^{2} \leq c \rho^{n-\mu}\left[\|\nabla u\|_{L^{2}(B)}^{2}+\|f\|_{L^{p}(B)}^{2}\right], \quad \forall y \in \bar{B}_{2}, \quad \forall \rho \leq R_{0} .
$$

Now set $y=x_{0}$ and $R_{0}=2$. In the rest of the proof we will denote $B_{r}:=B_{r}\left(x_{0}\right)$. By using standard covering argument if necessary, we obtain

$$
\int_{B_{r}}|\nabla u|^{2} \leq C r^{n-\mu}\left[\|\nabla u\|_{L^{2}(B)}^{2}+\|f\|_{L^{p}(B)}^{2}\right], \quad \forall r \leq 2 .
$$

On the other hand, for all $\rho<r \leq 2$,

$$
\begin{aligned}
\int_{B_{\rho}}\left|\nabla u-(\nabla u)_{\rho}\right|^{2} & \leq C\left[\left(\frac{\rho}{r}\right)^{n+2} \int_{B_{r}}\left|\nabla u-(\nabla u)_{r}\right|^{2}+\int_{B_{r}}|\nabla w|^{2}\right] \\
& \leq C\left[\left(\frac{\rho}{r}\right)^{n+2} \int_{B_{r}}\left|\nabla u-(\nabla u)_{r}\right|^{2}\right] \\
& +C\left(\|\nabla u\|_{L^{2}\left(B_{r}\right)}^{2} r^{2 \mu}+\|f\|_{L^{p}(B)}^{2} r^{n+2 \nu}\right)
\end{aligned}
$$

Combining (2.18) and (2.19) we conclude that $\nabla u \in C^{0, \gamma}\left(B_{1}\right), \gamma=\min (\nu, \mu / 2)$. In particular, as (2.16) in the proof of Theorem 2.1

$$
\sup _{B_{1}}|\nabla u| \leq C\left(\|\nabla u\|_{L^{2}\left(B_{2}\right)}+\|f\|_{L^{p}(B)}\right) \text {. }
$$

We may then use inequality (2.19) again for $\rho<r \leq 1$, getting

$$
[\nabla u]_{C^{0, \alpha}\left(B_{1}\right)} \leq C\left(\|\nabla u\|_{L^{2}(B)}+\|f\|_{L^{p}(B)}\right), \quad \alpha=\min (\mu, \nu) .
$$

This completes the proof.

Proof of Theorem 2.2. First, by Theorem 2.1 we know $u \in C_{l o c}^{0, \beta}(\Omega)$ for some $\beta>0$. Then $a(x):=\sigma(x, u(x))$ is locally Hölder continuous with some exponent $\gamma>0$. Hence, from Lemma 2.7 we conclude $\nabla u$ is locally Hölder continuous. In particular, $\nabla u$ is bounded in $B$. As in $(2.20)$ we have an estimate

$$
\sup _{B_{R / 2}}|\nabla u| \leq C\left(\|\nabla u\|_{L^{2}(B)}+\|f\|_{L^{p}(B)}\right) \text {. }
$$


Thus $a(x)$ is Hölder continuous in $B_{R / 2}$ with exponent $\mu$ and $[a]_{C^{0, \mu}\left(B_{R / 2}\right)} \leq K$, where $K$ is a constant depends on $\|\nabla u\|_{L^{2}(B)},\|f\|_{L^{p}(B)},[\sigma]_{\mu}$ and other prescribed quantities independent of $u, f$. Now, the theorem follows from Lemma 2.7.

Remark 2.3. In the proof of Theorem 2.1 we actually proved that if $f \in \mathcal{H}_{l o c}^{q / 2}\left(\Omega ; \mathbb{R}^{n}\right)$ and $g \in L_{l o c}^{q}\left(\Omega ; \mathbb{R}^{n}\right), q>n$, then any weak solution of the system

$$
\left.\begin{array}{c}
\nabla \times[a(x) \nabla \times u]=f+\nabla \times g \\
\nabla \cdot u=0
\end{array}\right\} \quad \text { in } \Omega
$$

satisfies the following estimate in $B:=B_{R}\left(x_{0}\right) \Subset \Omega$ :

$$
\|u\|_{C^{0, \alpha}\left(\bar{B}_{R / 2}\right)} \leq C\left[\|u\|_{L^{2}(B)}+\|f\|_{L^{q / 2}(B)}+\|g\|_{L^{q}(B)}\right] .
$$

Also, the proof of Lemma 2.7 implies that a weak solution of

$$
\left.\begin{array}{c}
\nabla \times[\sigma(x, u) \nabla \times u]=f+\nabla \times g \\
\nabla \cdot u=0
\end{array}\right\} \quad \text { in } \Omega,
$$

where $f \in \mathcal{H}_{l o c}^{p}(\Omega), p>n$, and $g \in C_{l o c}^{0, \beta}(\Omega), \beta>0$, is locally Hölder continuous with exponent $\alpha=\min (\mu, 1-n / p, \beta)$.

Remark 2.4. In two dimensional case, Hölder continuity of weak solutions of (2.1) may follow from Sobolev imbedding. In fact, if $f \equiv 0$, then a weak solution $u$ belongs to $W_{l o c}^{1, p}(\Omega)$ for all $p \in(1, \infty)$. However, when $n=3, C^{0, \alpha}$-regularity is the optimal result. To see this, consider a solution of the form $u=\left(0,0, u^{3}\right): \Omega \rightarrow \mathbb{R}^{3}$. Let us assume for simplicity $f \equiv 0$. Then, the system (2.1) becomes

$$
\left\{\begin{array}{l}
D_{3}\left(a(x) D_{1} u^{3}\right)=0 \\
D_{3}\left(a(x) D_{2} u^{3}\right)=0 \\
D_{1}\left(a(x) D_{1} u^{3}\right)+D_{2}\left(a(x) D_{2} u^{3}\right)=0 \\
D_{3} u_{3}=0
\end{array}\right.
$$

From the last equation of $(2.24)$, we can set $v\left(x_{1}, x_{2}\right):=u_{3}\left(x_{1}, x_{2}, x_{3}\right)$. It also follows that $a(x)$ depends only on $x_{1}$ and $x_{2}$. Then $v$ solves the following equation of divergence form in two variables:

$$
L v:=\sum_{i, j=1}^{2} D_{i}\left(a(x) D_{j} v\right)=0 \quad \text { in } \quad \Omega .
$$

The operator $L$ is called an isotropic operator. Piccinini \& Spagnolo showed that $v$ is locally Hölder continuous with exponent $\alpha=\frac{4}{\pi} \arctan \sqrt{m / M}$ (see [8] Theorem 2, page 396). To see that it is an optimal result, consult Example 2 of [8] on page 400 .

\section{Application to a Maxwell system}

As mentioned in the introduction, the problem we have analyzed so far arises from the Maxwell's system in a quasi-stationary electromagnetic field. Especially if the electric conductivity strongly depends on the temperature, then by taking the temperature effect into consideration the classical Maxwell system in a quasistationary electromagnetic field reduces to the following mathematical model (see 
[9], page 1029-1032):

$$
\left\{\begin{array}{l}
H_{t}+\nabla \times(\sigma(u) \nabla \times H)=0, \\
\nabla \cdot H=0, \\
u_{t}-\Delta u=\sigma(u)|\nabla \times H|^{2},
\end{array}\right.
$$

where $H$ and $u$ are unknowns representing, respectively, the strength of magnetic field and temperature while $\sigma(u)$ denotes the electric resistivity of the material which is assumed to be strictly positive and bounded; i.e. there exist positive numbers $m, M$ such that

$$
0<m \leq \sigma(s) \leq M, \quad \forall s \in \mathbb{R} .
$$

In [9], YIN proved, under appropriate assumptions on boundary and initial conditions, the existence of a pair of global weak solutions $(H, u)$ :

$$
\begin{aligned}
& H \in L^{\infty}\left(0, T ; L^{2}\left(\Omega ; \mathbb{R}^{3}\right)\right) \cap L^{2}\left(0, T ; W^{1,2}\left(\Omega ; \mathbb{R}^{3}\right)\right) \\
& u \in L^{\infty}\left(0, T ; L^{1}(\Omega ; \mathbb{R})\right) \cap L^{q}\left(0, T ; W^{1, q}(\Omega ; \mathbb{R})\right), \quad q \in[1,5 / 4) .
\end{aligned}
$$

In addition, he showed that if a pair of weak solutions $(H, u)$ are continuous, then they are classical provided that $\sigma$ is smooth enough. However, as pointed out by him, the continuity of weak solutions is unknown even if $\sigma$ is smooth. Continuity of weak solutions of (3.1) heavily relies on the regularity theory of the following system with bounded measurable coefficient $a(x, t)$ :

$$
\left.\begin{array}{c}
v_{t}+\nabla \times[a(x, t) \nabla \times v]=0 \\
\nabla \cdot v=0
\end{array}\right\} \quad \text { in } Q
$$

where $Q$ the space-time cylinder $\Omega \times(0, T)$ for some $T>0$. We don't know at this time whether or not weak solutions of the system (3.3) are Hölder continuous.

In this section, we consider instead the following fully steady-state systems introduced by YIN (see [9] page 1031):

$$
\left.\begin{array}{c}
\nabla \times(\sigma(u) \nabla \times H)=0 \\
\nabla \cdot H=0 \\
-\Delta u=\sigma(u)|\nabla \times H|^{2}
\end{array}\right\} \quad \text { in } \Omega .
$$

Using the results we obtained in previous section, we will show the $C^{0, \alpha}$-regularity of weak solutions of (3.4).

Theorem 3.1. Let $(H, u)$ be a pair of weak solutions of (3.4). Then $(H, u) \in$ $C_{\text {loc }}^{0, \alpha}(\Omega)$ for some $\alpha>0$. Moreover, the following estimates hold in $\Omega^{\prime} \Subset \Omega$ :

$$
[H]_{C^{0, \alpha}\left(\Omega^{\prime}\right)}+[u]_{C^{0, \alpha}\left(\Omega^{\prime}\right)} \leq C\left(m, M, \Omega^{\prime}, \Omega,\|H\|_{L^{2}},\|u\|_{L^{2}}\right) .
$$

Proof. Let $B:=B_{4 R}=B_{4 R}\left(x_{0}\right) \Subset \Omega$. We will show $(u, H)$ is Hölder continuous in $B_{R}=B_{R}\left(x_{0}\right)$. Indeed, from the proof of Theorem 2.1 we have

$$
\|H\|_{C^{0, \alpha}\left(B_{2 R}\right)} \leq C(m, M, R)\|H\|_{L^{2}(B)}, \quad \alpha=\alpha(m / M)>0 .
$$

It remains to show that $u$ is also Hölder continuous in $B_{R}$. Using a vector identity,

$$
\nabla \cdot(F \times G)=(\nabla \times F) \cdot G-F \cdot(\nabla \times G),
$$

together with the first equation $\nabla \times(\sigma(u) \nabla \times H)=0$ of (3.4), we obtain

$$
\nabla \cdot[H \times(\sigma(u) \nabla \times H)]=\sigma(u)|\nabla \times H|^{2} .
$$

We rewrite the last equation of (3.4) as follows:

$$
-\Delta u=\nabla \cdot[H \times(\sigma(u) \nabla \times H)] .
$$


As before, fix $r \leq R$ and split $u$ into two parts $v$ and $w:=u-v$ such that

$$
\left\{\begin{array}{c}
-\Delta v=0 \text { in } B_{r} \\
v=u \text { on } \partial B_{r} .
\end{array}\right.
$$

Then, as in (2.12), the following estimate holds for $\rho<r \leq R$ :

$$
\int_{B_{\rho}}\left|u-u_{\rho}\right|^{2} \leq C\left(\frac{\rho}{r}\right)^{n+2} \int_{B_{r}}\left|u-u_{r}\right|^{2}+C r^{2} \int_{B_{r}}|\nabla w|^{2} .
$$

We need to estimate $\|\nabla w\|_{L^{2}\left(B_{r}\right)}^{2}$. Since $w \in W_{0}^{1,2}\left(B_{r}\right)$ and satisfies

$$
-\Delta w=\nabla \cdot[H \times(\sigma(u) \nabla \times H)] \text { in } B_{r},
$$

integration by parts and Schwarz inequality yields

$$
\int_{B_{r}}|\nabla w|^{2} \leq 2 \int_{B_{r}} \sigma(u)^{2}|H|^{2}|\nabla \times H|^{2} \leq 2 M^{2} \int_{B_{r}}|H|^{2}|\nabla \times H|^{2} .
$$

Since $H$ is continuous, it is bounded in $B_{r}$ and thus from (3.11)

$$
\int_{B_{r}}|\nabla w|^{2} \leq C \sup _{B_{2 R}}|H|^{2} \int_{B_{r}}|\nabla \times H|^{2} .
$$

On the other hand, from the fact that $H$ solves the first equation of (3.4) it follows

$$
\int_{B_{r}}|\nabla \times H|^{2} \leq \frac{C}{r^{2}} \int_{B_{2 r}}\left|H-H_{2 r}\right|^{2} \leq C[H]_{C^{0, \alpha}\left(B_{2 R}\right)}^{2} r^{n-2+2 \alpha} .
$$

combining (3.12) and (3.13) together with (3.6) we obtain the required estimate:

$$
\|\nabla w\|_{L^{2}\left(B_{r}\right)}^{2} \leq C(m, M, R)\|H\|_{L^{2}(B)}^{4} r^{n-2+2 \alpha} .
$$

Finally, by inserting (3.14) into (3.10) we conclude from Lemma 2.6

$$
[u]_{C^{0, \alpha}\left(B_{R}\right)} \leq C(m, M, R)\left(\|u\|_{L^{2}(B)}+\|H\|_{L^{2}(B)}^{2}\right) .
$$

Now theorem follows from (3.6), (3.15) and standard covering argument.

Theorem 3.2. Let $(H, u)$ be a pair of weak solutions of (3.4). Assume further that $\sigma$ is Hölder continuous with exponent $\mu \in(0,1)$. Then $H \in C_{l o c}^{1, \mu}(\Omega)$ and $u \in C_{l o c}^{2, \mu}(\Omega)$.

Proof. First, by Theorem 2.1 we have $(u, H) \in C_{l o c}^{0, \alpha}(\Omega)$, which in turn implies $\sigma(u)$ is Hölder continuous with exponent $\beta=\alpha \mu$. Then $H \in C_{l o c}^{1, \beta}(\Omega)$ by Lemma 2.7 and thus $\sigma(u)|\nabla \times H|^{2} \in C_{l o c}^{0, \beta}(\Omega)$. Since $u$ solves

$$
-\Delta u=\sigma(u)|\nabla \times H|^{2} \quad \text { in } \Omega,
$$

it follows from the theory of Laplace operator that $u \in C_{l o c}^{2, \beta}(\Omega)$. In particular, $\nabla u$ is locally bounded and thus $\sigma(u) \in C_{l o c}^{0, \mu}(\Omega)$. By Lemma 2.7 again, $H \in C_{l o c}^{1, \mu}(\Omega)$. Therefore $\sigma(u)|\nabla \times H|^{2} \in C_{l o c}^{0, \mu}(\Omega)$ and $u \in C_{l o c}^{2, \mu}(\Omega)$ by (3.16). This completes the proof.

Remark 3.3. Let $(H, u)$ be a pair of weak solutions of (3.4). Suppose that $\sigma \in C^{k, \alpha}$ where $k$ is a nonnegative integer and $0<\alpha<1$. Then

$$
H \in C_{l o c}^{k+1, \alpha}(\Omega), \quad u \in C_{l o c}^{k+2, \alpha}(\Omega) .
$$

In particular, if $\sigma \in C^{1, \alpha}$ then $(H, u)$ is a pair of classical solutions. 


\section{REMARKS ON THE CASE $n \geq 4$}

First, we introduce some notations. Let $\Omega$ be a domain in $\mathbb{R}^{n}, n \geq 3$. Denote by $\Lambda^{k}:=\Lambda^{k}(\Omega)$ the class of $k$-forms in $\Omega$. Let $*: \Lambda^{k} \rightarrow \Lambda^{n-k}$ be the Hodge star linear operator, defined by setting

$$
*\left(d x^{i_{1}} \wedge \cdots \wedge d x^{i_{k}}\right)=\left(d x^{j_{1}} \wedge \cdots \wedge d x^{j_{n-k}}\right)
$$

and extending it linearly, where $\left(i_{1}, \cdots, i_{k}, j_{1}, \cdots, j_{n-k}\right)$ is an even permutation of $(1,2, \cdots, n)$ so that $d x^{i_{1}} \wedge \cdots \wedge d x^{i_{k}} \wedge d x^{j_{1}} \wedge \cdots \wedge d x^{j_{n-k}}=d$ vol. Let $d^{*}: \Lambda^{k} \rightarrow \Lambda^{k-1}$ be the adjoint of the exterior differential operator $d: \Lambda^{k-1} \rightarrow \Lambda^{k}$ with respect to the Hodge inner product:

$$
\langle\alpha, \beta\rangle:=\int_{\Omega} \alpha \wedge * \beta, \quad \text { where } \quad \alpha, \beta \in L^{2}\left(\Omega ; \Lambda^{k}\right) .
$$

More precisely, it is defined by $\langle d \alpha, \beta\rangle=\left\langle\alpha, d^{*} \beta\right\rangle$ for smooth forms $\alpha \in \Lambda^{k-1}(\Omega)$, $\beta \in \Lambda^{k}(\Omega)$, one of which with compact support in $\Omega$. From the Stoke's theorem, it follows that $d^{*}=(-1)^{n k+n+1} * d *$.

Let $u=\left(u^{1}, \cdots, u^{n}\right) \in W^{1,2}\left(\Omega ; \mathbb{R}^{n}\right)$. For the sake of simplicity, we will use the same notation $u$ for the corresponding 1 -form $\sum_{i=1}^{n} u^{i}(x) d x^{i}$. In this context, we denote its exterior differential $d u$ by

$$
d u:=\sum_{i<j}\left(D_{i} u^{j}-D_{j} u^{i}\right) d x^{i} \wedge d x^{j} .
$$

A celebrated result by De Giorgi [1] states that weak solutions to linear elliptic equations with $L^{\infty}$-coefficients are Hölder continuous. In contrast to this, as it is well known, weak solutions of linear elliptic equations with $L^{\infty}$-coefficients may have singularities. For example, De Giorgi [2] constructed a weak solution to an elliptic system with $L^{\infty}$-coefficients which belongs to $W^{1,2}\left(B_{1}(0) ; \mathbb{R}^{n}\right), n \geq 3$, but is not bounded.

Related to those results, Giaquinta \& Hong [4] raised an interesting question: Are weak solutions of the following system locally Hölder continuous?

$$
\left.\begin{array}{c}
d^{*}[a(x) d u]=0 \\
d^{*} u=0
\end{array}\right\} \quad \text { in } \Omega
$$

Here $a(x) \in L^{\infty}(\Omega)$ is assumed to be bounded by two positive numbers $m, M$. More generally, consider the following inhomogeneous system:

$$
\left.\begin{array}{rl}
d^{*}[a(x) d u] & =f+d^{*} g \\
d^{*} u & =0
\end{array}\right\} \quad \text { in } \quad \Omega
$$

where $f \in \mathcal{H}_{\text {loc }}^{p}\left(\Omega ; \mathbb{R}^{n}\right)$ and $g \in L_{\text {loc }}^{q}\left(\Omega ; \Lambda^{2}\right) \cong L_{l o c}^{q}\left(\Omega ; \mathbb{R}^{n(n-1) / 2}\right)$.

When $n=3$ the above system (4.3) is identical to the system (2.1) of Sec. 2 and Theorem 2.1 states the answer to their question is positive when $n=3$. However, our method used in the proof of Theorem 2.1 cannot be applied to the case when $n \geq 4$ and we don't know the answer in that case.

Let us briefly mention why the case $n=3$ is special. In the proof of Theorem 2.1 , we made use of the fact that de Rham cohomology of a ball $B \in \mathbb{R}^{n}$ is trivial in the sense that if $\alpha \in \Lambda^{2}(B)$ satisfies $d^{*} \alpha=0$ then there exists a $\beta \in \Lambda^{n-3}(B)$ such that $d \beta=* \alpha$. In the case when $n=3, \beta$ is a scalar function so that we may apply well-known result of De Giorgi [1] to get $C^{0, \alpha}$-estimate. 
The aim of this section is to compile known results from general theory of elliptic systems which can be applied to the system (4.3). We have the following identity similar to (2.4) (see e.g. [7], page 33):

$$
-\Delta \alpha=d^{*}(d \alpha)+d\left(d^{*} \alpha\right), \quad \forall \alpha \in \Lambda^{1}(\Omega) .
$$

Hence if $a(x)$ is continuous, then the perturbation method used in Lemma 2.7 can be applied here without any change. Also if the ratio, $M / m$ is sufficiently close to 1 , then it can be shown that weak solutions $u$ of the system (4.3) satisfy $u \in W_{l o c}^{1, p}$ for some $p>n$. Hölder continuity of $u$ will then follow from Sobolev imbedding.

We again emphasize that most of results in this section can be inferred from the general theories of elliptic systems, so we will provide proofs only when the situation is not quite obvious.

Proposition 4.1. Let $u \in W_{\text {loc }}^{1,2}\left(\Omega ; \mathbb{R}^{n}\right)$ be a weak solution of (4.3). Suppose $f \in$ $\mathcal{H}_{l o c}^{p}\left(\Omega ; \mathbb{R}^{n}\right), p>n / 2$ and $g \in L_{l o c}^{q}\left(\Omega ; \Lambda^{2}\right), q>n$. If $a(x)$ is continuous, then $u$ is locally Hölder continuous with exponent $\alpha=\alpha(n, m, M, p, q)>0$.

Proof. See Theorem 3.1 in [3] and following remark on page 87.

Proposition 4.2. Let $u \in W_{\text {loc }}^{1,2}\left(\Omega ; \mathbb{R}^{n}\right)$ be a weak solution of (4.3). Suppose $f \in$ $\mathcal{H}_{\text {loc }}^{q / 2}\left(\Omega ; \mathbb{R}^{n}\right)$ and $g \in L_{l o c}^{q}\left(\Omega ; \Lambda^{2}\right), q>n$. Then, there exists a number $\epsilon_{0}>1$ such that if $M / m<\epsilon_{0}$, then $\nabla u \in L_{\text {loc }}^{p}\left(\Omega ; \mathbb{R}^{n^{2}}\right)$ for some $p>n$. In particular, $u$ is locally Hölder continuous in $\Omega$.

Proof. The proof relies on the $L^{p}$ theory for Laplace operator and a perturbation argument (see e.g. [6] and Theorem 2.5 [page 154] in [3]).

Proposition 4.3. Let $u \in W_{\text {loc }}^{1,2}\left(\Omega ; \mathbb{R}^{n}\right)$ be a weak solution of (4.3). Assume $f \in$ $\mathcal{H}_{\text {loc }}^{p}\left(\Omega ; \mathbb{R}^{n}\right), p>n$ and $g \in C_{\text {loc }}^{0, \beta}\left(\Omega ; \Lambda^{2}\right), \beta>0$. If $a(x)$ is $C^{0, \mu}$-continuous, then $\nabla u$ is locally Hölder continuous with exponent $\alpha=\min (\mu, 1-n / p, \beta)$.

Proof. See Theorem 3.2 (page 88) in [3] and also Lemma 2.7 in Sec 2.

Lemma 4.1 (Caccioppoli-inequality). Let $u \in W_{l o c}^{1,2}\left(\Omega ; \mathbb{R}^{n}\right)$ be a weak solution of the system (4.3) with $f \in \mathcal{H}_{\text {loc }}^{2 n /(n+2)}\left(\Omega ; \mathbb{R}^{n}\right)$ and $g \in L_{\text {loc }}^{2}\left(\Omega ; \Lambda^{2}\right)$. Let $B_{R}:=$ $B_{R}\left(x_{0}\right) \Subset \Omega$. Then for any $\lambda \in \mathbb{R}^{n}$,

(4.5) $\int_{B_{R / 2}}|\nabla u|^{2} \leq C\left(\frac{1}{R^{2}} \int_{B_{R}}|u-\lambda|^{2}+\|f\|_{L^{2 n /(n+2)\left(B_{R}\right)}}^{2}+\|g\|_{L^{2}\left(B_{R}\right)}^{2}\right)$

where $C=C(n, m, M)$.

Sketch of proof. As in Lemma 2.2, there exists $h \in W_{0}^{1,2}\left(B_{R} ; \Lambda^{2}\right)$ such that

$$
f=d^{*} h \quad \text { in } \quad B_{R} ; \quad\|h\|_{L^{2}\left(B_{R}\right)} \leq C\|f\|_{L^{2 n /(n+2)}\left(B_{R}\right)} .
$$

Let $\eta \in C_{0}^{\infty}\left(B_{R} ; \mathbb{R}\right)$ be a cut-off function such that $0 \leq \eta \leq 1, \eta \equiv 1$ in $B_{R / 2}$ and $|\nabla \eta| \leq 4 / R$. By choosing $(u-\lambda) \eta^{2}$ as a test function it is easy to see

$$
\int_{B_{R}} \eta^{2}|d u|^{2} \leq C\left(\frac{1}{R^{2}} \int_{B_{R}}|u-\lambda|^{2}+\int_{B_{R}}|h|^{2}+\int_{B_{R}}|g|^{2}\right) .
$$

Since $d^{*} u=0$ in $\Omega$, (4.4) implies

$$
\begin{aligned}
\left\langle-\Delta u, \eta^{2}(u-\lambda)\right\rangle & =\left\langle d u, d\left(\eta^{2}(u-\lambda)\right)\right\rangle \\
& =\langle d u, 2 \eta d \eta \wedge(u-\lambda)\rangle+\left\langle d u, \eta^{2} d u\right\rangle .
\end{aligned}
$$


On the other hand, integration by parts yields

$$
\begin{aligned}
\left\langle-\Delta u, \eta^{2}(u-\lambda)\right\rangle & =\int_{\Omega} \nabla u \cdot \nabla\left(\eta^{2}(u-\lambda)\right) \\
& =\int_{\Omega} 2 \eta D_{j} u^{i} D_{j} \eta\left(u^{i}-\lambda^{i}\right)+\int_{\Omega} \eta^{2}|\nabla u|^{2} .
\end{aligned}
$$

Therefore,

$$
\int_{B_{R}} \eta^{2}|\nabla u|^{2} \leq C\left(\frac{1}{R^{2}} \int_{B_{R}}|u-\lambda|^{2}+\int_{B_{R}} \eta^{2}|d u|^{2}\right) .
$$

Combining (4.6), (4.7) and (4.8) we obtain (4.5).

Lemma 4.2 ( $L^{p}$-estimates). Suppose $f \in \mathcal{H}_{\text {loc }}^{q}\left(\Omega ; \mathbb{R}^{n}\right), q>2 n /(n+2)$ and $g \in$ $L_{l o c}^{r}\left(\Omega ; \Lambda^{2}\right), r>2$. Let $u \in W_{l o c}^{1,2}(\Omega)$ be a weak solution of the system (4.3). Then, $\nabla u \in L_{l o c}^{p}\left(\Omega ; \mathbb{R}^{n^{2}}\right)$ for some $p>2$. More precisely, let $B:=B_{R}\left(x_{0}\right) \Subset \Omega$, then

$$
\|\nabla u\|_{L^{p}\left(B_{R / 2}\right)} \leq C\left(\|\nabla u\|_{L^{2}(B)}+\|f\|_{L^{n p /(n+p)}(B)}+\|g\|_{L^{p}(B)}\right) .
$$

Sketch of proof. Let $h$ be as in (4.6). Setting $\lambda=(u)_{R}$ and then using SobolevPoincaré inequality, we obtain from (4.7) and (4.8)

$$
\oint_{B_{R / 2}}|\nabla u|^{2} \leq C\left[\left(\oint_{B}|\nabla u|^{s}\right)^{2 / s}+\oint_{B}|h|^{2}+\oint_{B}|g|^{2}\right], \quad s=\frac{2 n}{n+2} .
$$

It is so called reverse Hölder inequality. It is well known that higher integrability of $\nabla u$ follows from (4.10) (see e.g. Proposition 1.1 [page 122] of [3]). Also, as mentioned in Proposition 4.2, (4.9) can be derived by a perturbation argument based on the $L^{p}$-theory of Laplace operator.

With preceding lemmas at hand, let us consider the quasi-linear system:

$$
\left.\begin{array}{c}
d^{*}[\sigma(x, u) d u]=f+d^{*} g \\
d^{*} u=0
\end{array}\right\} \quad \text { in } \Omega,
$$

where $f \in \mathcal{H}_{l o c}^{p}(\Omega)$ and $g \in L_{l o c}^{q}\left(\Omega ; \Lambda^{2}\right)$.

By using general theory of elliptic systems, it is again more or less straightforward to show partial $C^{0, \alpha}$ (or $C^{1, \alpha}$ )-regularity for weak solutions of the system (4.11) under appropriate continuity assumptions on $\sigma$. We denote $k$-dimensional Hausdorff measure of $\Sigma \subset \mathbb{R}^{n}$ by $H^{k}(\Sigma)$.

Proposition $4.4\left(C^{0, \alpha}\right.$-partial regularity). Suppose $f \in \mathcal{H}_{l o c}^{q / 2}(\Omega)$ and $g \in L_{l o c}^{q}(\Omega)$, for some $q>n$ and let $u \in W_{\text {loc }}^{1,2}(\Omega)$ be a weak solution of the system (4.11). Assume that $\sigma$ is continuous. Then there exists an open set $\Omega_{0} \subset \Omega$ such that $u$ is locally Hölder continuous with exponent $1-n / q$ in $\Omega_{0}$. Moreover, $H^{n-s}\left(\Omega \backslash \Omega_{0}\right)=0$ for some $s>2$.

Proof. See Theorem 1.1 (page 166) in [3].

Proposition $4.5\left(C^{1, \alpha}\right.$-partial regularity). Suppose $\sigma$ is locally $C^{0, \alpha}$-continuous for some $\alpha \in(0,1)$. Let $u \in W_{\text {loc }}^{1,2}(\Omega)$ be a weak solution of the system (4.11) and let $f \in \mathcal{H}_{l o c}^{p}\left(\Omega ; \mathbb{R}^{n}\right), p=n /(1-\alpha), g \in C_{\text {loc }}^{0, \alpha}\left(\Omega ; \mathbb{R}^{n}\right)$. Then there exists an open set $\Omega_{0} \subset \Omega$ such that $u \in C_{l o c}^{1, \alpha}\left(\Omega_{0}\right)$ and $H^{n-s}\left(\Omega \backslash \Omega_{0}\right)=0$ for some $s>2$.

Proof. It follows from Proposition 4.5 and Lemma 2.7. 
Acknowledgment. We thank to Professor Min-Chun Hong and Professor Mariano Giaquinta for valuable communications. We also thank to Professor Mikhail Safonov and Professor Vladimír Šverák for helpful discussions.

\section{REFERENCES}

[1] De Giongi Sulla differenziabilità e l'analiticità delle estremali degli integrali multipli regolari, Mem. Accad. Sci. Torino. Cl. Sci. Fis. Mat. Nat. (3) 3 (1957), 25-43.

[2] De Giongi Un esempio di estremali discontinue per un problema variazionale di tipo ellittico, Boll. Un. Mat. Ital. (4) 1 (1968), 135-137.

[3] Mariano Giaquinta Multiple integrals in the calculus of variations and nonlinear elliptic systems, Annals of Mathematics Studies, 105, Princeton University Press, Princeton, NJ, 1983.

[4] M. Giaquinta \& M-C Hong Partial regularity of minimizers of a functional involving forms and maps, Preprint.

[5] Gilbarg \& Trudinger Elliptic partial differential equations of second order, 2nd ed., Springer-Verlag, Berlin, 1983.

[6] Norman G. Meyers An L $L^{p}$-estimate for the gradient of solutions of second order elliptic divergence equations, Ann. Scuola Norm. Sup. Pisa (3) 17 (1963) 189-206.

[7] Steven Rosenberg The Laplacian on a Riemannian manifold, Cambridge University Press, Cambridge, 1997.

[8] L. C. Piccinini \& S. Spagnolo On the Hölder continuity of solutions of second order elliptic equations in two variables, Ann. Scuola Norm. Sup. Pisa (3) 26 (1972), 391-402.

[9] Yin, Hong-Ming Regularity of solutions to Maxwell's system in quasi-stationary electromagnetic fields and applications, Comm. Partial Differential Equations 22 (1997), no. 7-8, 1029-1053.

[10] Yin, Hong-Ming Optimal regularity of solution to a degenerate elliptic system arising in Electromagnetic fields, Washington State University technical report 01-1.

School of Mathematics, University of Minnesota, Minneapolis, Minnesota 55455

E-mail address: kkang@math.umn.edu

E-mail address: skim@math.umn.edu 